\title{
Energy Transfer In the Core Plgments of Phycobillsomes from Mastigocladus laminosus As Studied by Temperature-Dependent Hole Burning
}

\author{
Alessandro Feis, ${ }^{*}+$ \\ Institut für Physikalische Chemie, J.-Gutenberg-Universität, D-6500 Mainz, Germany \\ Josef Friedrich, \\ Physikalisches Institut und Bayreuther Institut für Makromolekülforschung, Universität Bayreuth, \\ D-8580 Bayreuth, Germany
}

Lothar Gottschalk, and Hugo Scheer

Botanisches Institut, Universität München, D-8000 München 19, Germany (Received: January 6, 1992; In Final Form: April 1, 1992)

\begin{abstract}
Persistent optical hole burning has been performed in the allophycocyanin band of phycobilisomes of Mastigocladus laminosus. The homogeneous transition line width has been obtained by a careful analysis of the hole-burning data. The dependence of the line width on temperature has been measured in the range 1.5-4.2 K. A lifetime-limited value of $1.08 \pm 0.21 \mathrm{GHz}$ is obtained as a result of linear back extrapolation to $T=0 \mathrm{~K}$. It corresponds to an energy transfer time of $147 \pm 15$ ps from allophycocyanin to the terminal emitters of the phycobilisome core.
\end{abstract}

\section{Introduction}

Protein-protein and protein-cofactor interactions represent an attractive topic in the study of intermolecular interaction and biological function. Highly organized protein complexes play fundamental roles in living cells, such as molecular recognition, regulation of ionic fluxes or catalytic activity, gene regulation, etc. Photosynthetic systems are a representative example of organized protein complexes. They are built of various chromophore-binding proteins, which are arranged in a way to optimize light absorption, energy transfer, and energy conversion. It is a considerable advantage in the study of photosynthetic systems when the isolated subunit proteins are well characterized. In this respect, phycobilisomes (PBS), the light-harvesting complexes of cyanobacteria and red algae, are a profitable case. Isolation of PBS and of their constituent pigments, which are proteins containing bilin chromophores, has been achieved for many species. ${ }^{1}$ The main constituent proteins of PBS are phycocyanins, allophycocyanins, and phycoerythrins or phycoerythrocyanins, with relative abundance and aggregation states depending on the source, on the growth conditions, and on the isolation procedure. The physicochemical characterization of the proteins of PBS has been mainly performed by means of optical spectroscopy and ultracentrifugation studies. ${ }^{2}$ There is a growing number of known protein sequences, ${ }^{3}$ and X-ray structures have been determined for $C$-phycocyanin from several species ${ }^{4}$ and one phycoerythrocyanin. ${ }^{5}$

A comparison of the structural data with time-resolved spectroscopic experiments ${ }^{6,7}$ has allowed for elucidating the intra- and interprotein energy transfer processes following light absorption. In PBS of Mastigocladus laminosus, energy transfer is known to occur, on a time scale of tens of picoseconds, from $C$-phycocyanin to allophycocyanin (APC), and eventually to specialized long-wavelength-absorbing chromophores on APC-B and/or the large polypeptide(s). Owing to the complexity of the excitation decay process, time-resolved experiments produce decay curves which must be fitted with multiexponential laws. Persistent spectral hole burning, instead, offers the opportunity to probe the various steps one at a time. The site selectivity of the technique allows measurement of the homogeneous transition line widths of a selected group of chromophores. ${ }^{8}$ The determining energy

Present address: Dipartimento di Chimica Fisica, Università di Padova, I-35131 Padova, Italy. transfer time can then be determined for that group of chromophores, provided one main condition is fulfilled, that is, the contribution of pure dephasing to the homogeneous transition line width is absent. This situation is hypothetically reached at $T=$ $0 \mathrm{~K}$. Nevertheless, energy transfer times can be evaluated by back extrapolation to $0 \mathrm{~K}$ if the temperature dependence of the homogeneous line width is known. We therefore measured the homogeneous line width of APC chromophores in PBS of Mastigocladus laminosus in the temperature range $1.5-4.2 \mathrm{~K}$. Temperature-dependent data have been reported so far only for C-phycocyanin from Mastigocladus laminosus in a glass. ${ }^{9} \mathrm{Al}-$ though hole-burning spectra of PBS from the same source at 4.2 $\mathrm{K}$ have also been measured, ${ }^{10}$ the temperature dependence for the whole PBS assembly has not yet been determined.

\section{Experimental Section}

Low temperature glasses of energetically coupled phycobilisomes from Mastigocladus laminosus ${ }^{11}$ were prepared as described before. ${ }^{10}$ Absorption and fluorescence spectra were measured with a 1-m Jobin-Yvon monochromator. Hole-burning spectra were measured with a continuous wave $(\mathrm{CW})$ ring dye laser (Coherent CR-699), having a bandwidth of $<10 \mathrm{MHz}$ in single mode operation. Holes were burnt with power densities ranging from 1 to $470 \mu \mathrm{W} / \mathrm{cm}^{2}$ and irradiation times ranging from $1 \mathrm{~s}$ to 30 $\mathrm{min}$ (see Results). The holes were detected by scanning the laser over the burn wavelength and measuring the transmission of light through the sample. All measurements were performed in a ${ }^{4} \mathrm{He}$ bath cryostat. The temperature was kept constant within 0.01 $\mathrm{K}$.

\section{Results}

Figure 1 shows an absorption spectrum of PBS, taken at 4.2 $K$ in the wavelength range $600-700 \mathrm{~nm}$. The main peak at 635 $\mathrm{nm}$ and the broad band system with its maximum at $610 \mathrm{~nm}$ are due to the absorption of $C$-phycocyanin. The peak at $652 \mathrm{~nm}$ corresponds to the absorption maximum of APC. The small band at $670 \mathrm{~nm}$ may be ascribed to the low-energy chromophores of APC-B and the large linker polypeptide(s). ${ }^{12}$ Figure 1 shows also a fluorescence spectrum of the same sample at $4.2 \mathrm{~K}$. The weak bands at shorter wavelength (644 and $662 \mathrm{~nm}$ ) originate from $C$-phycocyanin and APC, whereas the outstanding band at 687 $\mathrm{nm}$ is the emission from the terminal emitter(s). Both spectra are comparable to those of ref 10 . The fluorescence spectra are 


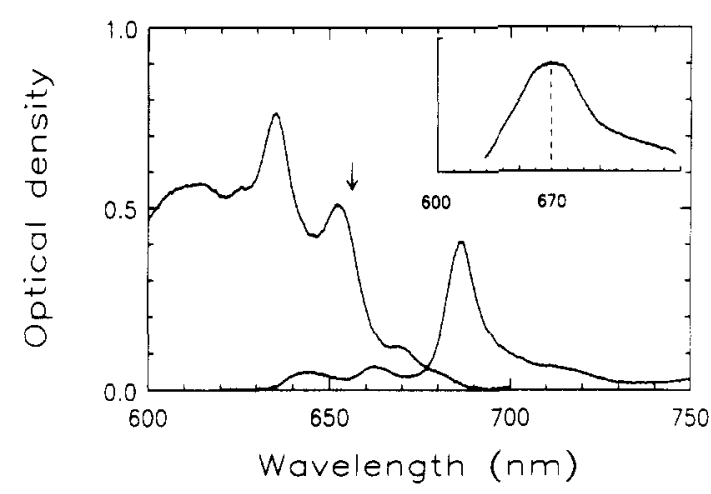

\begin{tabular}{l}
3 \\
3 \\
0 \\
0 \\
0 \\
5 \\
0 \\
0 \\
0 \\
0 \\
0 \\
2 \\
\hline
\end{tabular}

Figure 1. Absorption and fluorescence emission spectra of phycobilisomes from Mastigocladus laminosus at $4.2 \mathrm{~K}$. The arrow marks the wavelength by which hole burning was performed. (Insert) Fluorescence emission spectrum in "standard" conditions (see Results).

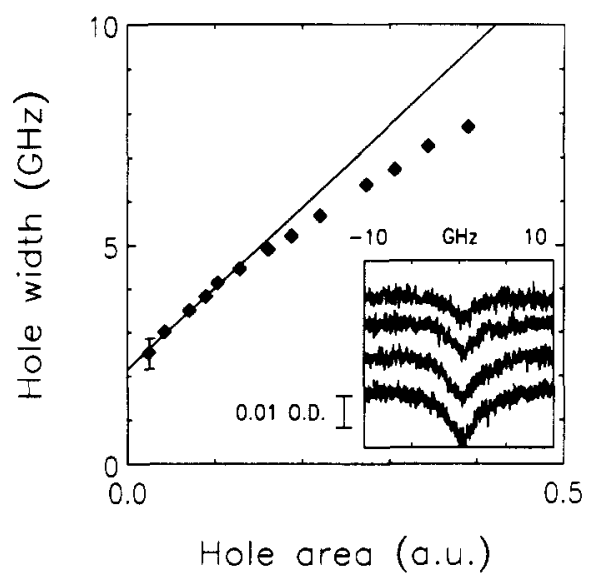

Figure 2. Hole width vs hole area in APC at $1.55 \mathrm{~K}$. The first six data points are linearly fitted (see Results). The first four points correspond to the hole-burning spectra shown in the insert, which are artificially scaled in order to make them legible.

strongly dependent on the optical density of the sample, since self-absorption of fluorescence alters both the position and the relative intensity of the peaks. We therefore measured the fluorescence spectra under standard conditions in order to test the quality of the samples, viz. room temperature and a concentration giving an optical density of 0.1 in a $1-\mathrm{cm}$ cuvette. A fluorescence spectrum of this kind is shown in the insert in Figure 1. The fluorescence spectra were independent of the exciting wavelength in the investigated range $(610-645 \mathrm{~nm})$. Intact PBS have maxima ranging from 670 to $676 \mathrm{~nm}$ under these standard conditions. A dissociation of PBS, leading to uncoupling of energy transfer, would be indicated by a blue shift of the emission maximum and the appearance of short-wavelength shoulders due to emission from free $C$-phycocyanin and APC.

We performed hole burning by irradiating the samples in the APC band at $657 \mathrm{~nm}$. In order to obtain accurate values for the homogeneous line widths, we carefully tried to avoid photochemical saturation broadening. This effect is due to the depletion of the ground-state population during the hole-burning process itself. ${ }^{13}$ Therefore, we measured the hole widths as a function of increasing irradiation times. By this means, we obtained the width for zero burning intensity through a back extrapolation. An example for such a series of measurements is given in the insert of Figure 2. The data points in Figure 2 show how the hole width evolves with the number of photoconverted centers as reflected in the hole area. A systematic feature of all our experiments was the early onset of saturation broadening.

The lifetime-limited hole width was obtained by performing a quasi-linear back extrapolation to $0 \mathrm{~K}$, using the line widths of the very shallow holes only. As it was shown previously, ${ }^{14}$ the slope of the extrapolation line of the width vs hole area is independent of temperature, as long as the Debye-Waller factor is independent of temperature. The results strongly support such

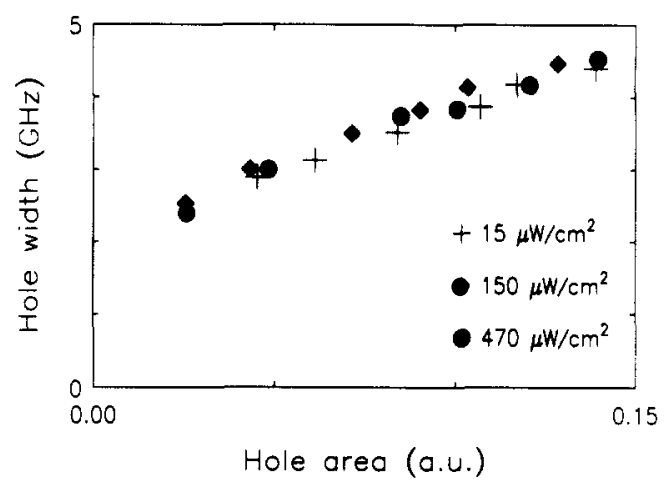

Figure 3. Hole width vs hole area in APC at $1.55 \mathrm{~K}$. Holes were burnt with different laser powers, namely $15(+), 150(\bullet)$, and $470(\bullet)$ $\mu \mathrm{W} / \mathrm{cm}^{2}$.

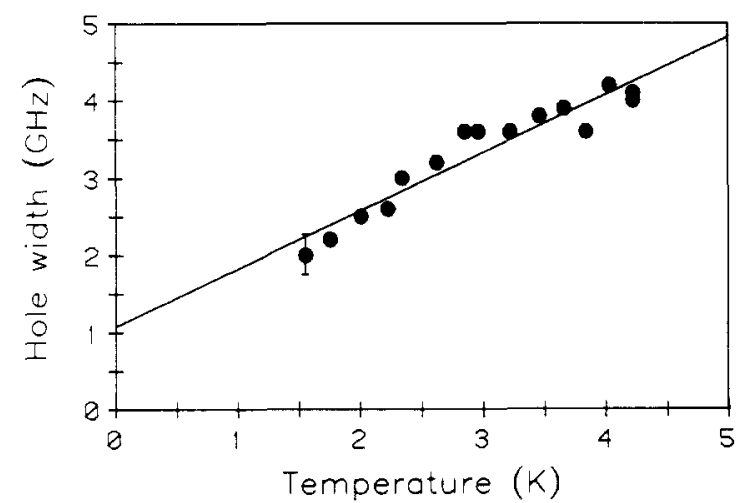

Figure 4. Temperature dependence of the hole width of APC at zero burning fluence. A linear fit is superimposed on the data.

a behavior. Therefore, we fitted all extrapolation data with a line having the same slope at all temperatures. This way, the lifetime limit of the hole-burning data should have a reasonable accuracy. We incidentally note that the larger width at $4.2 \mathrm{~K}$, which was observed in ref 10 , is possibly due to photochemical saturation broadening.

The laser power, too, is a possible source of extra broadening in the hole-burning process. This effect has a different origin as compared to photochemical saturation broadening. ${ }^{15}$ We experimentally proved the absence of power broadening by repeating the same series of measurements as in Figure 2 with laser power densities ranging from 15 to $470 \mu \mathrm{W} / \mathrm{cm}^{2}$ (see Figure 3). We found no evidence for an influence of power density on the hole width.

Figure 4 shows the temperature dependence of the hole width in the range $1.5-4.2 \mathrm{~K}$. Each value has been obtained by a back extrapolation to zero burning fluence, as shown in Figure 2. A linear fit is superimposed on the experimental data points. The lifetime-limited width has a value of $1.08 \pm 0.21 \mathrm{GHz}$.

\section{Discussion}

The results shown in Figures 2 and 3 rule out any sources of artificial hole broadening. On the other hand, a possible contribution to the hole width from spectral diffusion processes cannot be excluded. Spectral diffusion may occur on an extremely wide range of time scales. ${ }^{16}$ In particular, it may occur in the time between burning and reading the holes. Therefore, the extrapolated line width, as measured by spectral hole burning, is correctly referred to as quasihomogeneous. ${ }^{9}$ For PBS, it has been shown by temperature cycling experiments that spectral diffusion is not completely absent. ${ }^{17}$

If contributions from artificial broadening can be excluded, then the measured hole width $(\Delta \nu)_{\text {hole }}$ is just twice the homogeneous transition line width, $(\Delta \nu)_{\text {hom }}{ }^{13}$ plus the spectral diffusion width $(\Delta \nu)_{\mathrm{SD}}$

$$
(\Delta \nu)_{\text {hole }}=2(\Delta \nu)_{\text {hom }}+(\Delta \nu)_{\mathrm{SD}}
$$

The spectral diffusion width depends on temperature in a linear 
fashion (see, for instance, ref 16). The homogeneous width is related to energy relaxation time $\left(T_{1}\right)$ and pure dephasing time $\left(T_{2}^{*}\right)$ by the following equation

$$
2 \pi(\Delta \nu)_{\text {hom }}=\frac{1}{2 T_{1}}+\frac{1}{T_{2}^{*}}
$$

Since $1 / T_{2}{ }^{*}$, as well as $(\Delta \nu)_{\mathrm{SD}}$, vanishes as the temperature approaches zero, the measured line width can be related to $T_{1}$ in the limit $T=0 \mathrm{~K}$. Our experimental data allow an estimation of $T_{1}$. However, we have to assume a certain kind of temperature dependence of the line width. The temperature dependence of the homogeneous line width for solids at low temperature has been the subject of much theoretical and experimental work. ${ }^{16,18}$ Molecular mixed crystals exhibit an exponential temperature dependence, which originates from thermal excitation of local phonons. Most glasses have been shown to follow a quasi-linear temperature dependence, namely, a dependence on $T^{1.3}$. This has been correlated with a variety of models, all of which include coupling between the optical excitation and the structural defects in the glass. As far as data are available for proteins at low temperatures, they share many characteristics with amorphous systems. ${ }^{19}$ The temperature dependence of the homogeneous line width was well fitted by a $T^{1.16}$ law in the case of $C$-phycocyanin in a glass ${ }^{9}$ and by a $T^{1.27}$ law in the case of chlorophyllide apomyoglobin complex in a polymer. ${ }^{20}$ Line width data below $1 \mathrm{~K}$ are scarce. Recently, it was shown for a polymer that the width is dominated by spectral diffusion. ${ }^{21}$

If one assumes that the line width is spectral diffusion dominated in the temperature range of our experiment, a linear fit, as shown in Figure 4, is adequate. The back extrapolation to $T=0 \mathrm{~K}$ then allows estimation of $T_{1}$ of APC to be $147 \pm 15$ ps. The experimental data points might as well be fitted by a $T^{1.3}$ law, which does not significantly differ from the linear fit in the measured temperature range. In this case, the lifetime estimate would be $101 \pm 15 \mathrm{ps}$. It can be assumed that $T_{1}$ is almost totally determined by energy transfer processes. In fact, APC fluorescence is very low in PBS. We can also assume that it is the interprotein energy transfer which mainly determines the measured hole width, since (i) we burnt the red edge chromophores in the inhomogeneous band and (ii) intraprotein relaxation occurs on a much shorter time scale, 22 so it would show up in a much broader line width. A minor contribution to the observed $T_{1}$ might come from those chromophores of APC-B, which absorb at the same wavelength as those of APC, and which can directly transfer energy to their lower lying chromophores. The relaxation time has been determined for this couple of chromophores in APC-B from Mastigocladus laminosus by time-resolved fluorescence. It is on the order of $66 \mathrm{ps}$ at room temperature. ${ }^{22}$ Owing to the lesser quantity of these chromophores, we conclude that this contribution is negligible.

The interprotein energy transfer processes which take place in the core of PBS are directed from APC to two emitters, APC-B and/or the "anchor" protein. 23,24 The sequential path for energy transfer is still a matter of discussion. Time-resolved fluorescence measurements at $77 \mathrm{~K}^{7}$ in PBS from Anabaena variabilis support the idea that the pathways leading to the two terminal emitters are separated and originate from APC's with and without linker polypeptides, respectively. In our case, we cannot speculate on which of the two types of chromophores are selectively excited, but we can compare our result to the overall time for energy to flow from APC chromophores to the terminal emitters as observed in these measurements, i.e., about 100 ps. A very similar (120 ps) time constant was also observed ${ }^{25}$ for the energy transfer from APC to the terminal emitters in PBS from Mastigocladus laminosus at room temperature.

The analysis of ref 7 rests on the spatial model proposed by Glazer. ${ }^{26}$ An alternative model ${ }^{24}$ has been proposed, whereby excitation would be transferred from APC with linker to a complex containing the "anchor" protein, and, from this complex, to APC-B. In this case, the energy transfer time we determined should be mainly referred to the step: APC $\rightarrow$ "anchor".

\section{Conclusions}

We have shown that hole-burning spectroscopy allows the determination of single steps in the interprotein energy transfer of phycobilisomes, provided temperature-dependent data are obtained and artificial broadening sources can be excluded. We determined a time constant of $147 \mathrm{ps}$ for the energy transfer from allophycocyanin to the terminal emitters of PBS. This value is well consistent with fluorescence kinetic measurements.

Acknowledgment. This work has been supported by the Deutsche Forschungsgemeinschaft (SFB 262, Graduiertenkolleg "Nichtlineare Spektroskopie und Dynamik" [A.F., J.F.], SFB 143 [L.G., H.S.]) and the NATO-CNR Advanced Fellowship Program [A.F.].

\section{References and Notes}

(1) Bryant, D. A. In Light-energy Transduction in Photosynthesis: Higher Plant and Bacterial Models; Stevens, S. E. J., Bryant, D. A., Eds.; American Society of Plant Physiologists: Rockville, MD, 1988. Gantt, E. In Photosynthesis III-Pholosynthetic membranes and Light-Harvesting Systems; Staehelin, L. A., Arntzen, C. J., Eds.; Springer: Berlin, 1986. Scheer, H. In Phycobiliproteins: Molecular Aspects of Photosynthetic Antenna Systems; Fong, F. K., Ed.; Springer: Berlin, 1982. MacColl, R.; Guard-Friar, D. Phycobiliproteins: CRC Press: Boca Raton, FL, 1987. Wehrmeyer, W. In Proteins and nucleic acids in plant systematics; Jense, U., Fairbrother, D. E., Eds.; Springer: Berlin, 1983. Glazer, A. N. Annu. Rev. Biophys. Chem. 1985, 14, 47.

(2) See relevant articles in: Photosynthetic Light Harvesting Systems: Scheer, H., Schneider, S., Eds.; W. de Gruyter: Berlin, 1988.

(3) Anderson, L. K.; Grossman, A. R. J. Bacteriol. 1990, 172, 1289, 1297. Bryant, D. A. In ref 2, p 217. Sidler, W.; Zuber, H. In ref 2, p 49. Houmard, J.; Capuano, V.; Coursin, T.; Tandeau de Marsac, N. J. Bacteriol. 1988, 170, 5512.

(4) Dürring, M.; Schmidt, G. B.; Huber, R. J. Mol. Biol. 1991, 577 Schirmer, T.; Bode, W.; Huber, R. J. Mol. Biol. 1987, 196, 677. Schirmer, T.; Bode, W. J. Mol. Biol. 1986, 188, 651. Schirmer, T.; Bode, W.; Huber R.; Sidler, W.; Zuber, H. J. Mol. Biol. 1985, 184, 257.

(5) Dürring, M.; Huber, R.; Bode, W.; Rümbeli, R.; Zuber, H. J. Mol. Biol. 1990, 211, 633 .

(6) Sandström, A.; Gillbro, T.; Sundström, V.; Fischer, R.; Scheer, H. Biochim. Biophys. Acta 1988, 933, 42. Sandström, À.; Gillbro, T.; Sundström, V.; Wendler, J.; Holzwarth, A. R. Biochim. Biophys. Acta 1988, 933, 54 .

(7) Mimuro, M.; Yamazaki, I.; Tamai, N.; Katoh, T. Biochim. Biophys. Acta 1989, 973, 153 .

(8) Reddy, N. R. S.; Small, G. J.; Seibert, M.; Picorel, R. Chem. Phys. Lett. 1991, I81, 391. Johnson, S. G.; Small, G. J. J. Phys. Chem. 1991, 95 , 471. Tang, D.; Johnson, S. G.; Jankowiak, B.; Hayes, J. M.; Small, G. J.; Tiede, D. M. In 22nd Jerusalem Symposium: Perspectives in Photosynthesis; Jortner, J., Pullman, B., Eds.; Kluwer: Dordrecht, 1990. Gillie, J. K.; Small, G. J.; Golbeck, J. H. J. Phys. Chem. 1989, 93, 1620. Johnson, S. G.; Small, G. J. Chem. Phys. Lett. 1989, 155, 371. Gillie, J. K.; Hayes, J. M.; Small, G. J. Golbeck, J. H. J. Phys. Chem. 1987, 91 , 5524. van der Laan, H.; Schmidt, Th.; Visschers, R. W.; Visscher, K. J.; van Grondelle, R.; Völker, S. Chem. Phys. Lett. 1990, $170,231$.

(9) Köhler, W.; Friedrich, J.; Fischer, R.; Scheer, H. Chem. Phys. Lett. $1988,146,280$.

(10) Köhler, W.; Friedrich, J.; Fischer, R.; Scheer, H. J. Chem. Phys. $1988,89,871$

(11) Nies, M.; Wehrmeyer, W. Planta 1980, 150, 330.

(12) Reuter, W.; Wehrmeyer, W. Arch. Microbiol. 1988, 150, 534

(13) Friedrich, J.; Haarer, D. Angew. Chem. 1984, 96, 96. Angew. Chem., Int. Ed. Engl. 1984, 23, 113 .

(14) Breinl, W.; Friedrich, J.; Haarer, D. Phys. Rev. 1986, B34, 7271.

(15) Shen, Y. R. The Principles of Nonlinear Optics; Wiley: New York, 1984.

(16) Narasimhan, L. R.; Littau, K. A.; Pack, D. W.; Bai, Y. S.; Elschner, A.; Fayer, M. D. Chem. Rev, 1990, 90, 439.

(17) Köhler, W.; Friedrich, J. J. Chem. Phys. 1989, 90, 1270.

(18) Volker, S. Annu. Rev. Phys. Chem. 1989, 40, 499. Hayes, J. M.; Jankowiak, R.; Small, G. J. In Persistent Spectral Hole-Burning. Science and Applications; Moerner, W. E., Ed.; Springer: Berlin, 1988. Skinner, J. L.; Hsu, D. Adv. Chem. Phys. 1986, 65, 1.

(19) Friedrich, J. Mol. Cryst. Liq. Cryst. 1990, 183, 91

(20) Boxer, S. J.; Gottfried, D. S.; Lockhart, D. J.; Middendorf, T. R. J. Chem. Phys. 1987, 86, 2439.

(21) Müller, K. P.; Haarer, D. Phys. Rev. Lett. 1991, 66, 2344.

(22) Holzwarth, A. R.; Bittersmann, E;; Reuter, W.; Wehrmeyer, W. Biophys. J. 1990, 57, 133.

(23) Mimuro, M.; Lipschultz, C.; Gantt, E. Biachim. Biophys. Acta 1986, $852,126$.

(24) Reuter, W.; Wehrmeyer, W. Arch. Microbiol. 1990, 153, 111.

(25) Fischer, R. Doctoral Thesis, Universităt München, 1991.

(26) Glazer, A. N. Biochim. Biophys. Acta 1984, 768, 29. 